\title{
Two Approaches to the Ratio of the Charm Structure Functions
}

\author{
G.R.Boroun*i \\ Physics Deparetment, Razi University, Kermanshah, Iran \\ E-mail: borounerazi.ac.ir
}

\begin{abstract}
We show that the ratio of the charm structure functions with respect to the approaches according to the gluon distribution function behavior is approximately independent of $\mathrm{x}$ and the details of the parton distribution function at NLO analysis. These behaviors are according to the hard-Pomeron behavior and expanding methods at low $\mathrm{x}$ analysis. It is shown that the charm structure functions obtained are in agreement with data from HERA and other methods.
\end{abstract}

The XIth International Conference on Heavy Quarks and Leptons,

June 11-15, 2012

Prague, Czech Republic

* Speaker.

${ }^{\dagger}$ A footnote may follow. 


\section{Introduction}

In perturbative quantum Cheromodynamic calculations, the production of heavy quarks at HERA proceeds dominantly via the direct Boson-gluon fusion where the photon interacts with a gluon from the proton by the exchange of a heavy quark pair. With respect to the recent measurements of HERA, the charm contribution to the structure function at small $x$ is a large fraction of the total, as this value is approximately $30 \%$ fraction of the total [1]. This behavior is directly related to the growth of the gluon density at small $x$, as gluons couple only through the strong interaction. Consequently, the gluons are not directly probed in DIS, only contributing indirectly is via the $g \rightarrow q+\bar{q}$. This involves the computation of the BGF process $\gamma^{*}+g \rightarrow c+\bar{c}$. In this work, we study the charm structure functions behavior with respect to the gluon distribution behavior at NLO analysis. Then we present the ratio of the charm structure functions, which is independent of the gluon distribution.

\section{Mathematical Formalism}

The deeply inelastic charm structure functions in the cross section is given by [2]

$$
F_{k}^{c}\left(x, Q^{2}, m_{c}^{2}\right)=2 e_{c}^{2} \frac{\alpha_{s}\left(\mu^{2}\right)}{2 \pi} \int_{1-\frac{1}{a}}^{1-x} d z C_{g, k}^{c}(1-z, \zeta) \times G\left(\frac{x}{1-z}, \mu^{2}\right),
$$

where $a=1+4 \zeta\left(\zeta \equiv \frac{m_{c}^{2}}{Q^{2}}\right), G\left(x, \mu^{2}\right)$ is the gluon distribution function and $\mu$ is the mass factorization scale, which has been put equal to the renormalization scales $\mu^{2}=4 m_{c}^{2}$ or $\mu^{2}=4 m_{c}^{2}+Q^{2}$. Here $C_{g, k}^{c}$ is the charm coefficient functions in LO and NLO analysis as

$$
C_{k, g}(z, \zeta) \rightarrow C_{k, g}^{0}(z, \zeta)+a_{s}\left(\mu^{2}\right)\left[C_{k, g}^{1}(z, \zeta)+\bar{C}_{k, g}^{1}(z, \zeta) \ln \frac{\mu^{2}}{m_{c}^{2}}\right]
$$

where $a_{s}\left(\mu^{2}\right)=\frac{\alpha_{s}\left(\mu^{2}\right)}{4 \pi}$ and in the NLO analysis

$$
\alpha_{s}\left(\mu^{2}\right)=\frac{4 \pi}{\beta_{0} \ln \left(\mu^{2} / \Lambda^{2}\right)}-\frac{4 \pi \beta_{1}}{\beta_{0}^{3}} \frac{\ln \ln \left(\mu^{2} / \Lambda^{2}\right)}{\ln \left(\mu^{2} / \Lambda^{2}\right)}
$$

with $\beta_{0}=11-\frac{2}{3} n_{f}, \beta_{1}=102-\frac{38}{3} n_{f}$ ( $n_{f}$ is the number of active flavours). In the LO analysis, the coefficient functions BGF can be found [3], as

$$
\begin{aligned}
C_{g, 2}^{0}(z, \zeta)= & \frac{1}{2}\left(\left[z^{2}+(1-z)^{2}+4 z \zeta(1-3 z)-8 \zeta^{2} z^{2}\right] \times \ln \frac{1+\beta}{1-\beta}+\beta[-1+8 z(1-z)\right. \\
& -4 z \zeta(1-z)])
\end{aligned}
$$

and

$$
C_{g, L}^{0}(z, \zeta)=-4 z^{2} \zeta \ln \frac{1+\beta}{1-\beta}+2 \beta z(1-z)
$$

where $\beta^{2}=1-\frac{4 z \zeta}{1-z}$. At NLO, $O\left(\alpha_{e m} \alpha_{s}^{2}\right)$, the contribution of the photon- gluon component is usually presented in terms of the coefficient functions $C_{k, g}^{1}, \bar{C}_{k, g}^{1}$. Using the fact that the virtual 
photon- quark(antiquark) fusion subprocess can be neglected, because their contributions to the heavy-quark leptoproduction vanish at LO and are small at NLO. In a wide kinematic range, the contributions to the charm structure functions in NLO are not positive due to mass factorization. Therefore the charm structure functions are dependence to the gluonic observable in LO and NLO. The NLO coefficient functions are only avaliable as computer codes. But in the high- energy regime $(\zeta<<1)$ we can used the compact form of these coefficients according to the Ref.[4].

\section{The Hard- Pomeron behavior Method}

The small- $x$ region of the DIS offers a unique possibility to explore the Regge limit of PQCD. HERA data for the charm structure functions require only a hard pomeron as the coupling of the soft pomeron to charm is apparently very small. Exploiting the low-x behavior of the gluon distribution function according to the hard (Lipatov) pomeron as

$$
G\left(x, \mu^{2}\right) \rightarrow x^{-\lambda_{g}} .
$$

The power of $\lambda_{g}$ is found to be either $\lambda_{g} \simeq 0$ or $\lambda_{g} \simeq 0.5$. The first value corresponds to the soft Pomeron and the second value to the hard (Lipatov) Pomeron intercept. Based on the hard (Lipatov) pomeron behavior for the gluon distribution, let us put Eq.(3.1) in Eq.(2.1). After doing the integration over $z$, Eq.(2.1) can be rewritten as [5]

$$
F_{k}^{c}\left(x, Q^{2}, m_{c}^{2}\right)=2 e_{c}^{2} \frac{\alpha_{s}\left(\mu^{2}\right)}{2 \pi} I_{k}\left(x, \mu^{2}\right) \times G\left(x, \mu^{2}\right),
$$

where

$$
I_{k}\left(x, \mu^{2}\right)=\int_{1-\frac{1}{a}}^{1-x} C_{g, k}^{c}(1-z, \zeta)(1-z)^{\lambda_{g}} d z
$$

here $C_{g, k}^{c}$ is defined by Eq.2.2. In fact, the gluon distribution function input $G\left(x, \mu^{2}\right)$ does cancels in the ratio of the charm structure functions as we have [5]

$$
R^{c}=\frac{I_{L}\left(x, \mu^{2}\right)}{I_{2}\left(x, \mu^{2}\right)} .
$$

Therefore, this ratio, which is independent of the gluon distribution function, is very useful for practical applications. In this equation we used the solutions of the NLO BGF analysis and considered $\lambda_{g}$ as a hard (Lipatov) Pomeron.

\section{The Expanding Method}

Now we want to calculate the charm structure functions by using the expansion method for the gluon distribution function [6]. As can be seen, the dominant contribution to the charm structure functions comes from the gluon density at small $x$, regardless of the exact shape of the gluon 
distribution. The argument $\frac{x}{1-z}$ of the gluon distribution in Eq.2.1 can be expanded at an arbitrary point $z=\alpha$ as

$$
\left.\frac{x}{1-z}\right|_{z=\alpha}=\frac{x}{1-\alpha} \sum_{k=1}^{\infty}\left[1+\frac{(z-\alpha)^{k}}{(1-\alpha)^{k}}\right]
$$

The above series is convergent for $|z-\alpha|<1$. Using this expression we can rewrite and expanding the gluon distribution $G\left(\frac{x}{1-z}\right)$ as [6]

$$
G\left(\frac{x}{1-z}\right)=G\left(\frac{x}{1-\alpha}\right)+\frac{x}{1-\alpha}(z-\alpha) \frac{\partial G\left(\frac{x}{1-a}\right)}{\partial x}+O(z-\alpha)^{2} .
$$

Retaining terms only up to the first derivative in the expansion and doing the integration, we obtain our master formula as

$$
F_{k}^{c}\left(x, Q^{2}, m_{c}^{2}\right)=2 e_{c}^{2} \frac{\alpha_{s}\left(\mu^{2}\right)}{2 \pi} A_{k}(x) \times G\left(\frac{x}{1-\alpha}\left(1-\alpha+\frac{B_{k}(x)}{A_{k}(x)}\right)\right),
$$

where

$$
A_{k}(x)=\int_{1-\frac{1}{a}}^{1-x} C_{g, k}^{c}(1-z, \zeta) d z
$$

and

$$
B_{k}(x)=\int_{1-\frac{1}{a}}^{1-x}(z-\alpha) C_{g, k}^{c}(1-z, \zeta) d z .
$$

where $C_{g, k}^{c}$ is defined at Eq.2.2 in LO and NLO analysis and also $\alpha$ has an arbitrary value $0 \leq \alpha<1$. Eq.4.3 can be rewritten as [6]

$$
F_{k}^{c}\left(x, Q^{2}, m_{c}^{2}\right)=2 e_{c}^{2} \frac{\alpha_{s}\left(\mu^{2}\right)}{2 \pi} \eta_{k} G\left(\frac{x}{1-\alpha}\left(\beta_{k}-\alpha\right), \mu^{2}\right) .
$$

This result shows that the charm structure functions $F_{k}^{c}\left(x, Q^{2}\right)$ at $x$ are calculated using the gluon distribution at $\frac{x}{1-\alpha}\left(\beta_{k}-\alpha\right)$. Therefore, the gluon distribution at $\frac{x}{1-\alpha}\left(\beta_{k}-\alpha\right)$ can be simply extracted the charm structure functions $\left(F_{2}^{c}\right.$ and $\left.F_{L}^{c}\right)$ in the low $x$ values according to the coefficients at the limit $x \rightarrow 0$, in Table 1 at Ref.[6]. Moreover, there is a directly relation between the charm structure functions and gluon distribution via the well known Bethe- Heitler process $\gamma^{\star} g \rightarrow c \bar{c}$.

Now, we defining the ratio of the charm structure functions and obtain the following equation

$$
R^{c}=\frac{\eta_{L}}{\eta_{2}} \frac{G\left(\frac{x}{1-\alpha}\left(\beta_{L}-\alpha\right)\right.}{G\left(\frac{x}{1-\alpha}\left(\beta_{2}-\alpha\right)\right.} .
$$

We observe that the right-hand side of this ratio is independent of $x$ and independent of the gluon distribution input according to the coefficients in Table 1 at Ref.6. As in the low $x$ range we have

$$
R^{c} \approx \frac{\eta_{L}}{\eta_{2}}
$$

which is very useful to extract the charm structure function $F_{2}^{c}\left(x, Q^{2}\right)$ from measurements of the doubly differential cross section of inclusive deep inelastic scattering at DESY HERA, independent of the gluon distribution function. 


\section{Results and Conclusion}

We present the numerical results [5,6] for both two methods and compared our results with the experimental data (H1 Collab. [7]) and other methods [8-10]. In conclusion, these methods are useful to extract the charm structure function from the reduced charm cross section. The agreement between our predictions with the results obtained by $\mathrm{H} 1$ Collaboration using a more accurate but rather cumbersome procedure is remarkably good. Also the ratio of the charm structure functions is independent of $\mathrm{x}$ and independent of the parton distribution function input.

\section{References}

[1] K.Lipka, PoS (EPS-HEP)313, 2009.

[2] M.Gluk, E.Reya and A.Vogt, Z.Phys.C67,433 (1995).

[3] M.Gluk, E.Reya and A.Vogt, Eur.Phys.J.C5,461 (1998) .

[4] A. Y. Illarionov, B. A. Kniehl and A. V. Kotikov, Phys. Lett. B 663, 66 (2008).

[5] G.R.Boroun and B.Rezaei, Nucl.Phys.B857, 143(2012).

[6] B.Rezaei and G.R.Boroun, JETP 142, N0.3, 1(2012).

[7] F.D.Aaron, et.al., [H1 Collab.], Eur.Phys.J.C65,89(2010); Eur.Phys.J.C71,1509 (2011).

[8] N.N. Nikolaev, V.R. Zoller, Phys. Lett. B 509 (2001) 283.

[9] A.Donnachie and P.V.Landshoff, Phys.Lett.B 550, 160(2002).

[10] M.M.Block, L.Durand and D.W.Mckay, Phys.Rev.D77, 094003(2008). 

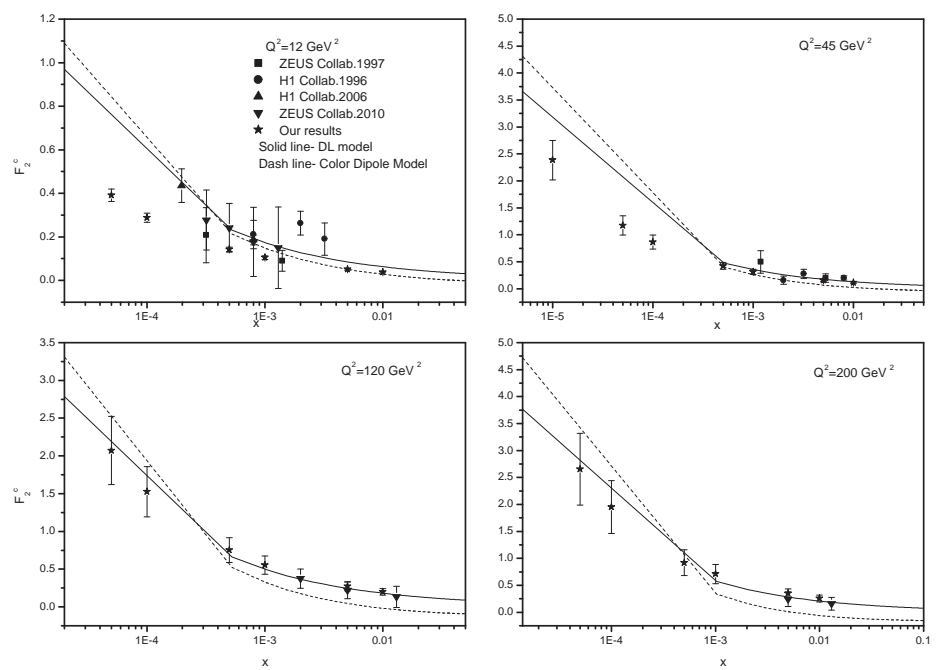

Figure 1: The charm component of the structure function [5] for different values of $Q^{2}(12,45,120$ and $200 \mathrm{GeV}^{2}$ ) as function of $x$. These results are the NLO predictions that accompanied to the theoretical uncertainty related to the renormalization scales. The solid and dash curves represents $F_{2}^{c}$ for DL [3-5] and color dipole [25] models. Data are from H1 and ZEUS Collab. that accompanied to the total errors [15].
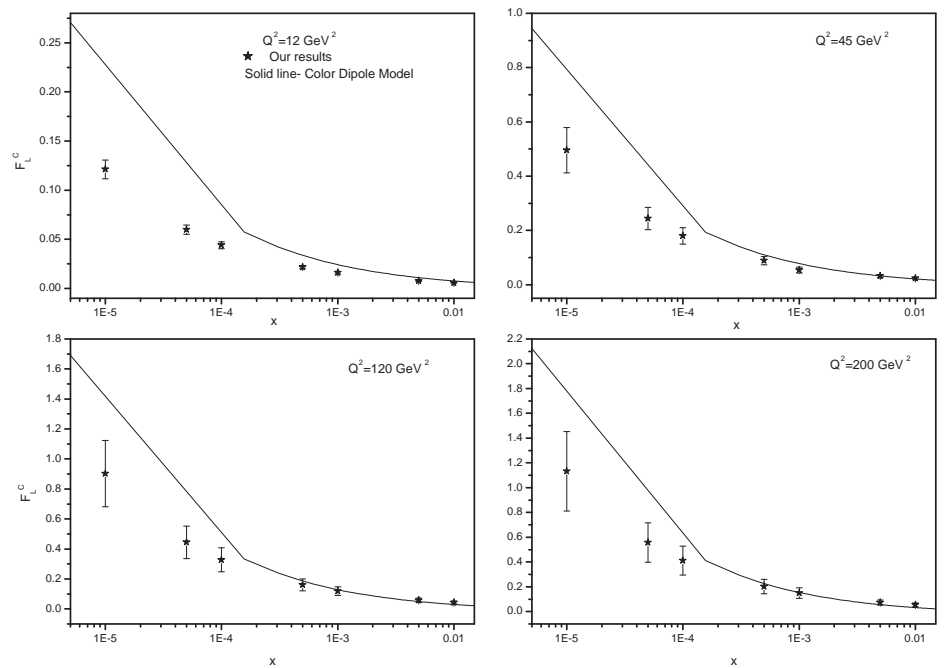

Figure 2: The charm component of the longitudinal structure function [5] at the $Q^{2}$ values $12,45,120$ and $200 \mathrm{GeV}^{2}$ as function of $x$. These results are the NLO predictions that accompanied to the theoretical uncertainty related to the renormalization scales. The solid curves represents $F^{c}{ }_{L}$ from the color dipole [25] model. 

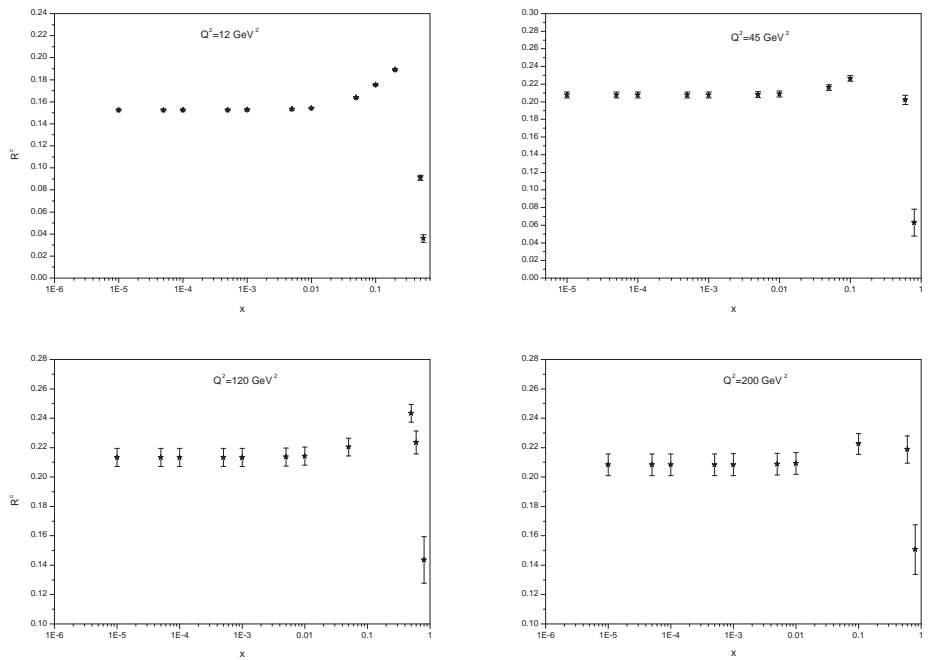

Figure 3: The ratio $R^{c}=F_{L}^{c} / F_{2}^{c}$ [5] as a function of $x$ for different values of $Q^{2}$ in NLO analysis.

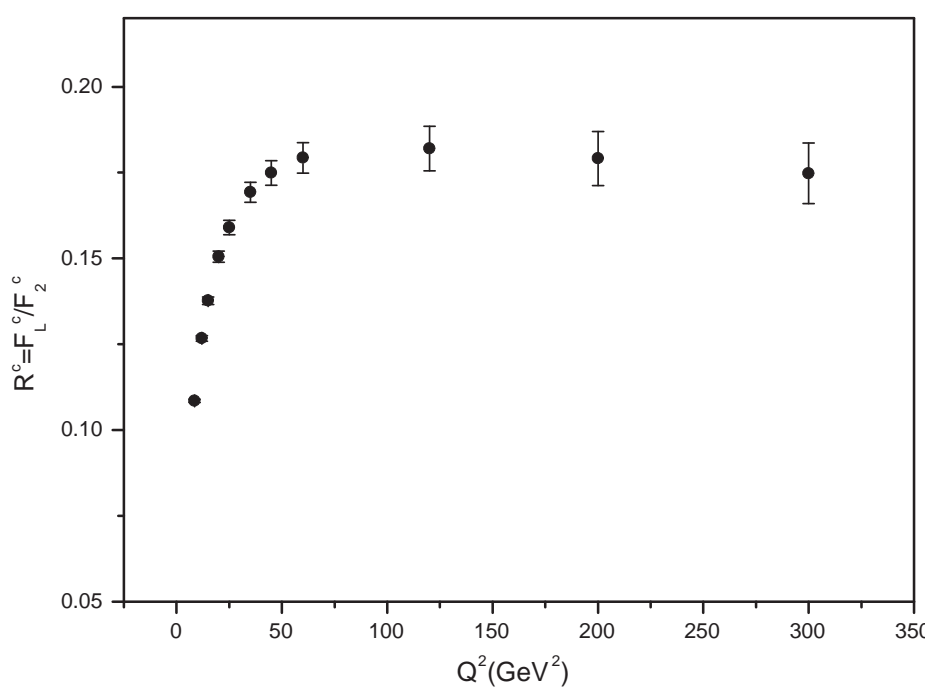

Figure 4: The ratio $R^{c}$ evaluated [6] as function of $Q^{2}$ at NLO analysis from Eq.16. The error bars are the theoretical uncertainty using the renormalization scales $\mu^{2}=4 m_{c}^{2}$ and $\mu^{2}=4 m_{c}^{2}+Q^{2}$. 


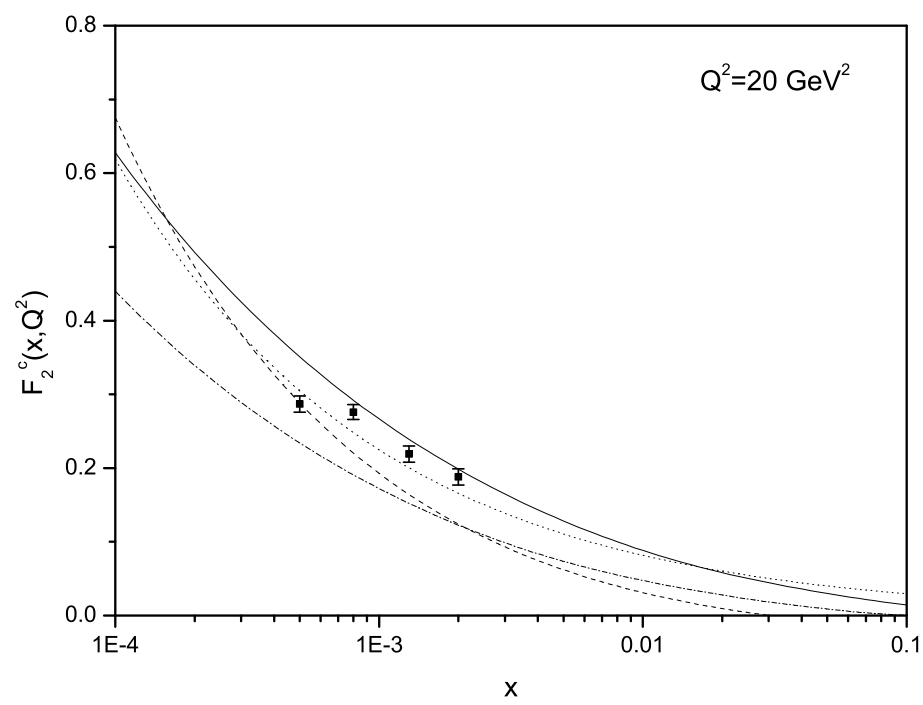

Figure 5: The charm structure function $\left(F_{2}^{c \bar{c}}\right)$ obtained [6] at $Q^{2}=20 \mathrm{GeV}^{2}$ with respect to the input gluon distribution NLO-GRV parametermization [9] (Solid line according to the expanding point $\alpha=0$ and DashDot line according to the expanding point $\alpha=0.8$ ) compared with DL fit[17-19] (Dot line), color dipole model [20] (Dash line) and H1 data [13] (square) that accompanied with total errors at the renormalization scale $\mu^{2}=4 m_{c}^{2}+Q^{2}$.

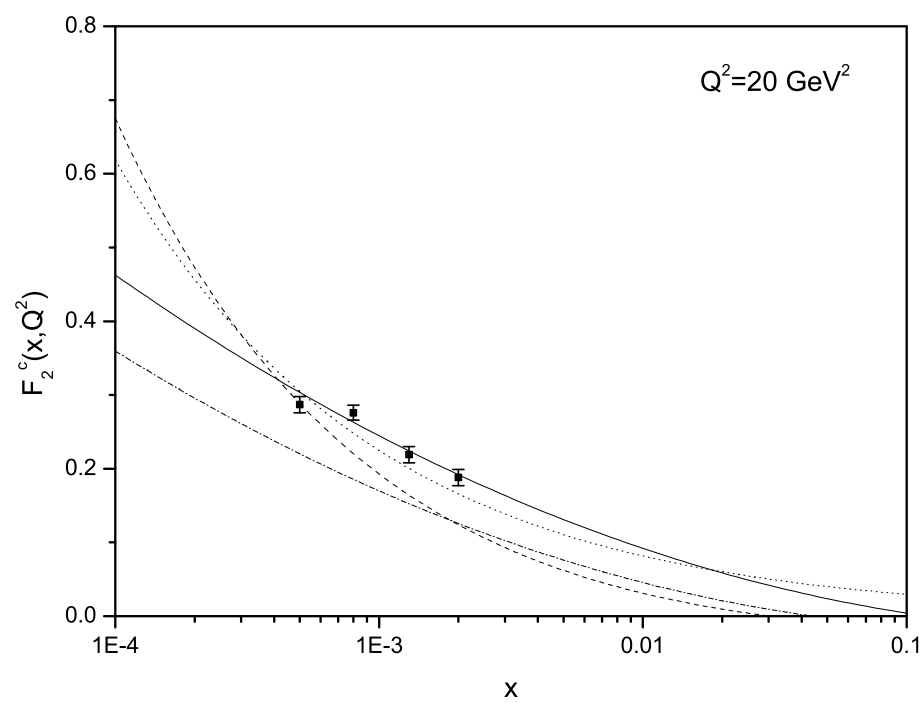

Figure 6: The charm structure function $\left(F_{2}^{c \bar{c}}\right)$ obtained [6] at $Q^{2}=20 \mathrm{GeV}^{2}$ with respect to the input gluon distribution Block fit [16] (Solid line according to the expanding point $\alpha=0$ and Dash-Dot line according to the expanding point $\alpha=0.8$ ) compared with DL fit[17-19] (Dot line), color dipole model [20] (Dash line) and $\mathrm{H} 1$ data [13] (square) that accompanied with total errors at the renormalization scale $\mu^{2}=4 m_{c}^{2}+Q^{2}$. 